\title{
Effect of CYP2D6 polymorphisms on plasma concentration and therapeutic effect of risperidone
}

Jinjun $\mathrm{Lu}^{1 \dagger}$, Ye Yang ${ }^{2 \dagger}$, Jian $\mathrm{Lu}^{1}$, Zuqing Wang ${ }^{1}$, Yiping $\mathrm{He}^{1}$, Yeliang Yan ${ }^{1}$, Kai Fu${ }^{1}$, Wenjuan Jiang ${ }^{1}$, Yunqing Xu', Renrong $\mathrm{Wu}^{2^{*}} \mathbb{D}$, Wenqing Liu ${ }^{1^{*}}$ and Jingping $\mathrm{Zhao}^{2}$

\begin{abstract}
Background: This study aimed to investigate the influence of CYP2D6 polymorphisms on risperidone plasma concentrations in patients with schizophrenia. Based on pharmacogenomics, we examined whether plasma concentration of risperidone is associated with clinical response and adverse side-effects.
\end{abstract}

Methods: We recruited patients with chronic schizophrenia who were then treated with risperidone. The CYP2D6 genotypes were determined using targeted sequencing. All high-frequency mutation sites of the nine exons of the gene were assayed in the present study. Plasma concentrations of risperidone and 9-hydroxyrisperidone (9-OH-RIS) were measured using high-performance liquid chromatography (HPLC). Psychiatric symptoms were monitored using The Positive and Negative Syndrome Scale (PANSS), Brief Psychiatric Rating Scale (BPRS), and Clinical Global Impression (CGI). Adverse effects were evaluated using the Barnes Akathisia Scale (BAS) and Extrapyramidal Symptom Rating Scale (ESRS). Follow-up visits were scheduled at weeks 2,4, and 8 after treatment initiation.

Results: Among the 76 patients, 100 C > T (rs1065852), 1038 C > T (rs1081003), 1662 G > C (rs1058164), 2851 C > T (rs16947), and 4181G > C (rs1135840) variants were detected. The most common allele was CYP2D6*10 (81.6\%), whereas CYP2D6*2 (9.2\%) and CYP2D6*5 (17.1\%) were relatively rare. Plasma levels of risperidone and the risperidone/9-OH risperidone ratio $(\mathrm{R} / 9-\mathrm{OH})$ were significantly increased in individuals with CYP2D6*10 $(P<0.05)$. The change in PANSS score, weight, high-density lipoprotein (HDL) level, prolactin (PRL) level, and ESRS were significantly different from baseline, between the different genotypes $(P<0.01)$. Moreover, individuals with CYP2D6*10 homozygous (T) mutations were associated with higher risperidone concentration and R/9-OH ratio than those with heterozygous mutations $(C T)(P<0.01)$. A change from baseline in BPRS scores was observed only during week 8 and was different between heterozygous and homozygous mutations. As for the C2851T polymorphism, the incidence of adverse metabolic effects was significantly different between the $C / C$ and $C / T$ genotypes $(P<0.01)$. Regarding the G4181C polymorphisms, the changes from baseline in GLU and TG, were different between the $\mathrm{C} / \mathrm{C}$ and $\mathrm{C} / \mathrm{G}$ genotypes $(P<0.01)$.

(Continued on next page)

\footnotetext{
* Correspondence: wurenrong@csu.edu.cn; maolv520a@sina.com

${ }^{\dagger}$ Jinjun Lu and Ye Yang contributed equally to this work.

${ }^{2}$ National Clinical Research Center for Mental Disorders, and Department of

Psychiatry, The Second Xiangya Hospital of Central South University,

Changsha 410011, Hunan, China

'The Third People's Hospital of Jiangyin City, Wuxi, Jiangsu Province, China
}

\section{$\triangle B M C$}

(c) The Author(s). 2021 Open Access This article is licensed under a Creative Commons Attribution 4.0 International License, which permits use, sharing, adaptation, distribution and reproduction in any medium or format, as long as you give appropriate credit to the original author(s) and the source, provide a link to the Creative Commons licence, and indicate if changes were made. The images or other third party material in this article are included in the article's Creative Commons licence, unless indicated otherwise in a credit line to the material. If material is not included in the article's Creative Commons licence and your intended use is not permitted by statutory regulation or exceeds the permitted use, you will need to obtain permission directly from the copyright holder. To view a copy of this licence, visit http://creativecommons.org/licenses/by/4.0/. The Creative Commons Public Domain Dedication waiver (http://creativecommons.org/publicdomain/zero/1.0/) applies to the data made available in this article, unless otherwise stated in a credit line to the data. 
(Continued from previous page)

Conclusions: The genotype of CYP2D6 significantly influences the plasma concentration of risperidone and may subsequently influence the adverse side-effects following risperidone treatment, while also exerting a slight influence on clinical outcomes.

Keywords: CYP2D6, Polymorphisms, Risperidone, Plasma concentration, Therapeutic effect

\section{Background}

Risperidone, an atypical antipsychotic, is widely used for the treatment of psychiatric problems and is associated with better compliance and reduced incidence of adverse reactions compared with typical antipsychotics [1]. Although risperidone has shown to be more effective relative to other antipsychotics, there are still some patients with psychiatric problems who do not respond to any antipsychotic therapeutics [2, 3]. Such situations can lead to patients having persistent exposure to antipsychotic drugs, which can be accompanied by serious side-effects, increased discomfort, and ultimately resulting in poor treatment adherence $[4,5]$. Among different patients, considerable clinical heterogeneity has been observed in the effects of risperidone, which affects the efficacy of treatment for mental illness [6]. Understanding the range of treatment responses and limiting risperidone exposure to within the therapeutic range, are important factors for achieving optimal treatment outcomes.

Risperidone is predominantly metabolized by the cytochrome P450 enzymes CYP2D6 and CYP3A4 [7]. The gene encoding CYP2D6 exhibits high polymorphism, and allelic variants are considered to influence the metabolic pathway of antipsychotics [8]. The main active metabolite of risperidone is 9-hydroxyrisperidone (9-OHRIS), which exhibits similar pharmacological actions to risperidone [9]. Therefore, it is suggested that the therapeutic response of risperidone can be influenced by the actions of plasma risperidone and the concentration of 9-OH-RIS [6]. Indeed, there is emerging evidence highlighting the heterogeneity of plasma concentrations of risperidone and 9-OH-RIS, as well as treatment response, between patients with different CYP2D6 genotypes $[10,11]$. Significantly higher risperidone levels and risperidone/9-OH-RIS(R/9-OH) ratios have been reported in patients carrying the CYP2D6*10/*10 variant compared with other alleles [12]. Similar results have been reported for children with autism spectrum disorders treated with risperidone, in that those with the CYP2D6*5/*10, CYP2D6*10/*10, or CYP2D6*10/*41 alleles exhibited higher levels of risperidone after administration [13]. These observations indicate that specific CYP2D6 variants influence the pharmacokinetics of risperidone. Further, an apparent association has been reported between CYP2D6 polymorphisms and clinical improvement in response to risperidone. Specifically, significant clinical improvements, assessed via the Positive and Negative Syndrome Scale - Thai (PANSS-T), have been reported in patients with the CYP2D6 "poormetabolizer" phenotype compared with treatment outcomes in "extensive" metabolizers [14]. However, this result has not been verified and the conclusions are still largely controversial. A previous study involving 136 patients with psychotic disorders treated with a single-dose of risperidone, did not identify any significant association between CYP2D6 polymorphisms and clinical recovery [15], which was supported by another study involving female patients with schizophrenia that found that PANSS improved following risperidone treatment, but was not associated with CYP2D6 genotype [16]. These studies highlight the discrepancies in the literature relating to CYP2D6, which may originate from the different genotypes that were analyzed in different studies. To the best of our knowledge, there have been no comprehensive studies exploring the effects of CYP2D6 variations on clinical outcomes of risperidone treatment.

Adverse effects, in particular metabolic-related effects, can contribute to reduced patient compliance irrespective of symptomatic improvement [17]. The risk of adverse metabolic effects in response to risperidone has been shown to vary greatly between individuals [11], indicating that genetics might play a significant role in susceptibility to adverse metabolic effects, indeed, CYP2D6*10 has been reported to be significantly associated with weight gain after risperidone treatment [17]. Other adverse side-effects, such as elevated prolactin and extrapyramidal syndrome (EPS), have also been reported in association with particular CYP2D6 variants $[18,19]$. However, in adult populations, the association between CYP2D6 polymorphisms and serum prolactin concentrations are unclear and whether this association exists in patients with schizophrenia, remains to be investigated. Discrepancies have also been reported regarding the relationship between CYP2D6 polymorphisms and EPS, with several studies failing to detect any significant difference in the incidence of EPS in association with CYP2D6 variations, while some studies reported only a marginally significant trend [20, 21]. The effects of CYP2D6 polymorphism on EPS in response to risperidone are unclear and require further exploration. 
It is clear that genotypic differences contribute to the discrepancies observed in clinical outcomes. We detected all mutations in nine exons of the full-length CYP2D6 sequence, which was further investigated in order to obtain a more comprehensive understanding of CYP2D6 polymorphisms. The present study aimed to investigate whether CYP2D6 polymorphisms significantly effect serum/plasma concentrations of risperidone in a clinical setting. To this end, we used pharmacogenomics to investigate whether plasma concentrations of risperidone are associated with clinical responses and the incidence of adverse effects, including adverse metabolic effects and EPS.

\section{Methods}

\section{Patients and methods}

We recruited patients that were all between 18 to 68 years of age and had been diagnosed with chronic schizophrenia (according to the criteria of the International Classification of Diseases-Tenth Edition [ICD10]), within the last 5 years, at Third People's Hospital of Jiangyin City, Jiangsu Province between May 2018, and May 2019. All patients had to meet the following criteria: 1) had not taken any antipsychotics drugs for at least 1 year; 2) had an acute attack and were re-admitted to the hospital; and 3) were treated with risperidone monotherapy after hospitalization. Risperidone dosage was increased gradually from a low dose to a therapeutic dose within 1 week (3-6 mg/day). Exclusion criteria were as follows: 1) Had undergone electroconvulsive therapy within 3 months of study enrollment, 2) administration of other psychotropic medications, including antidepressants and mood stabilizers, 3) had obvious brain damage or serious physical illness, and 4) presented with any other condition that rendered the patient unsuitable for the trial (e.g., allergies to related drugs, enrollment in clinical trials for other drugs or devices within 3 months of starting of the study). In total, 79 patients were recruited in the current study; however, 3 patients were dropped out of the study owing to drug adjustment or non-compliance. Ultimately, 76 patients were enrolled for subsequent data analyses.

We collected data at the time of enrollment. Baseline data included demographic information, a comprehensive medical history, clinical evaluation of psychiatric symptoms, a physical examination (including weight and height), laboratory tests (including fasting low-density lipoprotein [LDL-C], triglyceride, cholesterol, highdensity lipoprotein [HDL-C], glucose levels, prolactin levels, liver and renal function tests, and electrocardiography), and analysis of CYP2D6 genotype. The PANSS, Brief Psychiatric Rating Scale (BPRS), and Clinical Global Impression (CGI) were employed to monitor psychiatric symptoms. Treatment outcome was measured by assessing the change in clinical-symptom-scale scores over the study period. The improvement of clinical symptoms was expressed by the changes on the PANSS, BPRS, CGI scale scores from the initial assessment (week 0) to week 4 and then at week 8. The Barnes Akathisia Scale (BAS) and Extrapyramidal Symptom Rating Scale (ESRS) were used to evaluate adverse effects. Follow-up visits were made at 2,4 and 8 weeks after the initiation of treatment. During each follow-up visit, all evaluations (the physical examination, laboratory tests, weight measurements, clinical symptoms, and assessments of adverse effects) were repeated. Additionally, the concentrations of risperidone and 9-OH-RIS were measured at each follow-up visit.

\section{Evaluation of plasma concentration of risperidone}

Fasting blood samples were collected from patients at each follow-up visit. Blood draws were consistently performed between 7 and $8 \mathrm{am}$, to ensure consistency across patients. Plasma was stored at $-20^{\circ} \mathrm{C}$ until analysis. Plasma concentrations of risperidone and 9-OHRIS were determined using high-performance liquid chromatography (HPLC) with the Agilent 1260 High Performance Liquid Chromatograph (Agilent 1260 HPLC, USA). The ratio of risperidone to 9-OH-RIS (R/ 9-OH ratio) was calculated from the concentration of risperidone divided by the concentration of 9-OH-RIS (ng/mL) to reflect the activity of CYP2D6. The concentration/dose $(\mathrm{C} / \mathrm{D})$ ratio was calculated by dividing the total concentration of risperidone plus 9-OH-RIS (ng/ $\mathrm{mL}$ ) by risperidone dose $(\mathrm{mg} /$ day), to provide an index of drug elimination capacity.

\section{Genotyping}

An additional 3-4 $\mathrm{mL}$ volume of blood was drawn once for pharmacogenetic analyses at baseline. The whole blood sample was stored at $-70^{\circ} \mathrm{C}$ for subsequent extraction of genomic DNA. CYP2D6 genotypes were determined in all the patients using PCR assays followed by targeted sequencing. CYP2D6 has nine exons that were all assayed in the current study. The whole experimental process consisted of primer design, polymerase chain reaction (PCR), and sequencing. Four sets of upstream and downstream primers were designed with Oligo6 software to amplify the nine exons using genomic DNA as templates. Extraction of DNA was carried out using the YC-B nucleic acid extraction reagent (Hygeianecy Biological Company, Wuhan, China) according to manufacturer's instructions. The DNA was extracted quickly then adsorbed onto a high-performance solidphase matrix to obtain high-purity DNA by elution. The quality of genomic DNA underwent strict quality control including Nanodrop quantification and 1\% agarose gel electrophoresis. The details of individual PCR programs 
were summarized in Supplemental Table 1. Finally, the 3730xl DNA Analyzer was used for sequencing (Applied Biosystems, USA). Only the five high-frequency mutation sites of the nine exons were detected, including $100 \mathrm{C}>\mathrm{T}$ (rs1065852), 1038C $>\mathrm{T} \quad$ (rs1081003), 1662G $>\mathrm{C}$ (rs1058164), 2851C > T mutations (rs16947), and 4181G >C mutations (rs1135840). The frequencies of mutations at the different sites are detailed in Table 1 . The different mutation sites included 100C > T:62 (33TT,29CT), 1038C > T:62 (24TT,38CT), 1662C > G:68 (36CC,32CG), 2851C > T:20 (1TT,19CT), and 4181G > C:71 (43CC,28GC). These sites were categorized into CYP2D6*10, CYP2D6*2, and CYP2D6*65 according to the genotyping criteria for CYP2D6 (Pharmacogene Variation Consortium, https:// www.pharmvar.org/gene/CYP2D6).

\section{Statistical analysis}

Statistical analyses were carried out using the Statistical Package for Social Sciences (SPSS version 23, Chicago, IL, USA). Two-tailed $p$-values of $<0.05$ were considered to be of statistical significance. Continuous data with normal distribution are presented as mean and standard deviation, while non-normally distributed data are

Table 1 Demographic characteristics and genotypes of the study population

\begin{tabular}{ll}
\hline & Total study population $(\boldsymbol{n}=\mathbf{7 6})$ \\
\hline Sex (male/female) & $38 / 38$ \\
Age (years) & $45.0(33.0,54.0)$ \\
Height (cm) & $164.5(158.0,171.0)$ \\
Weight (kg) & $65.5(56.3,74.5)$ \\
BMl & $23.3(21.4,27.9)$ \\
Dose (mg) & $5.0(4.0,6.0)$ \\
PANSS & $88.0(82.0,95.0)$ \\
BPRS & $45.5(41.3,50.8)$ \\
CGI-S (2 week) & $5.0(5.0,6.0)$ \\
CGS-I (2 week) & $3.0(3.0,4.0)$ \\
Mutation sites & \\
$100(C>T)$ & $62(81.6)$ \\
$1038(C>T)$ & $62(81.6)$ \\
$1662(G>C)$ & $68(89.5)$ \\
$2851(C>T)$ & $20(26.3)$ \\
$4181(G>C)$ & $71(93.4)$ \\
Genotypes & \\
CYP2D6*10 & $77(81.6)$ \\
CYP2D6*2 & $7(9.2)$ \\
CYP2D6*65 & $13(17.1)$ \\
\hline
\end{tabular}

Data are presented as median (interquartile range) or number (\%) Abbreviation: BMI Body mass index, PANSS Positive and Negative Syndrome Scale, BPRS Brief Psychiatric Rating Scale, CGI-S Clinical Global ImpressionSeverity, CGI-I Clinical Global Impression-Improvement presented as median and interquartile range. Analysis of variance was used to compare data with normal distribution, while data with a non-normal distribution were analyzed using a 2-tailed Mann-Whitney U test to compare between two groups, or the Kruskal-Wallis test to compare multiple groups. The chi-square test was used to test differences in the distribution of categorical variables. Further comparisons between groups were carried out using the Dunn-Bonferroni post-hoc test. Multiple linear regression analysis was performed to analyze the relationship between $C Y P 2 D 6$ genotype and clinical response.

\section{Results}

\section{Demographic and genotypic characteristics}

The mean course of disease for chronic patients was approximately $13.48( \pm 7.80)$ years. The study sample was comprised of 76 patients. Demographic and clinical data of the study population are summarized in Table 1 . The risperidone dose ranged from 2 to $6 \mathrm{mg}$ among the study population. All subjects completed the clinical symptom evaluations including PANSS, BPRS and CGI at baseline. All results are presented in Table 1.

The majority of patients carried the CYP2D6*10 allele. The $1038 \mathrm{C}>\mathrm{T}(\mathrm{rs} 1081003)$ and $1662 \mathrm{G}>\mathrm{C}(\mathrm{rs} 1058164)$ mutations were nonsense mutations; therefore, they were excluded from subsequent analysis of single nucleotide polymorphisms. Given that the CYP2D6*10 allele is the most common allelic mutation, the heterozygous and homozygous mutations were further analyzed in our study.

Relationships between genotype and drug concentration, clinical outcome, and adverse effects

Plasma concentration of risperidone in different genotypes

Figure 1 illustrates the plasma concentrations of risperidone, at each follow-up time point, in relation to genotype. Plasma/genotype interactions were significantly different between genotypes at each time point (all $P<$ 0.05). The Kruskal-Wallis Test revealed an apparent association between CYP2D6 genotype and plasma concentration of risperidone (all $P<0.05$ ). Further pairwise comparisons revealed that, at all time points, plasma concentration of risperidone was significantly higher in subjects with the CYP2D6*10 genotype compared with those with the CYP2D6*2 genotype, after Bonferroni correction (all $P<0.05$ ). Moreover, the plasma concentrations of risperidone in patients with the CYP2D6*65 and $C Y P 2 D 6 * 2$ genotypes were significantly different at week $8(Z=2.627, P=0.026)$, while no significant differences were observed between subjects with the CYP2D6*65 and CYP2D6*10 alleles (all $P>0.05$ ). We did not observe any difference in 9-OH-RIS between genotypes (all $P>0.05$ ). The $\mathrm{R} / 9-\mathrm{OH}$ ratio was significantly higher in subjects with the CYP2D6*10 allele than those 

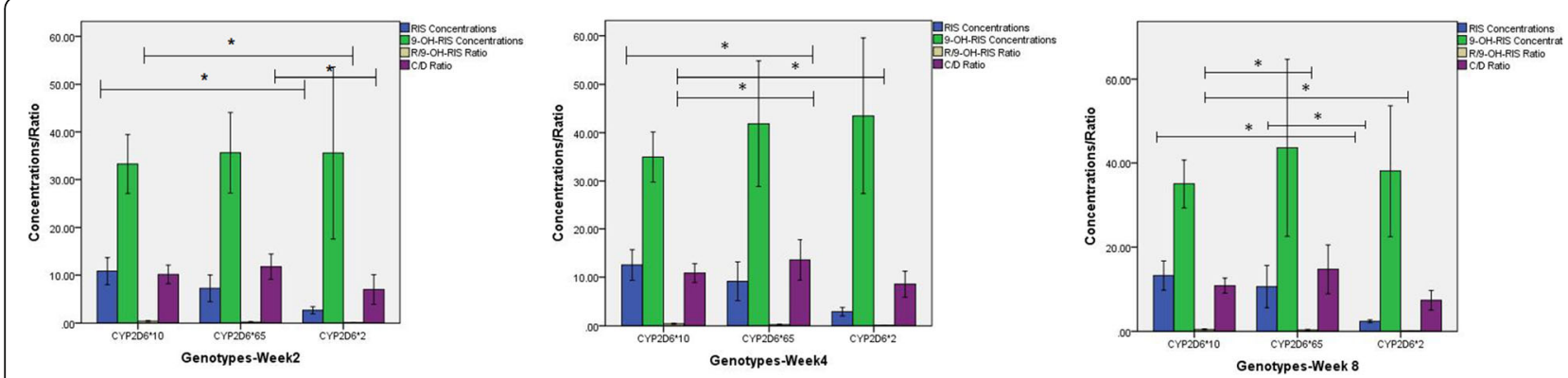

Fig. 1 Bar graphs showing plasma concentrations of risperidone in relation to genotype. Abbreviations: C/D, concentration/dose ratio; RIS, risperidone; 9-OH-RIS, 9-hydroxyrisperidone; R/9-OH-RIS Ratio: risperidone/9-hydroxyrisperidone ratio; ${ }^{*} p<0.05$

with the $C Y P 2 D 6 * 2$ allele, at all time points, and those with the CYP2D6*65 allele at 4 and 8 weeks (all $P<0.05$ after correction). The $\mathrm{C} / \mathrm{D}$ ratio at 2 weeks was significantly different between subjects with the CYP2D6 genotype $(Z=7.126, P=0.028)$, with a significant difference observed between CYP2D6*65 and CYP2D6*2 genotypes $(Z=2.642, P=0.025)$; however, the strength of this correlation decreased over time with no significant association observed at 4 or 8 weeks (both $P>0.05$ ).

\section{Relationship between polymorphisms of CYP2D6 and response to treatment}

Symptom improvement in relation to genotype is presented in Table 2. The change of PANSS score from 0 to 8 weeks was significantly different between $C Y P 2 D 6$ genotypes $(F=3.850, P=0.027)$. PANSS scores were significantly higher in individuals with the $C Y P 2 D 6 * 2$ allele than in those with the CYP2D6*65 allele after pairwise comparison using Bonferroni's post-hoc analysis $(P=$ 0.007). CYP2D6*10 was also higher than CYP2D6*65; however, the difference was not statistically significant $(P=0.052)$. Additionally, no significant difference was observed between CYP2D6*10 and CYP2D6*2 ( $P=$ 0.112). The BPRS and CGI scores were not significantly different among different genotypes from baseline to any follow-up visit (all $P>0.05$ ).

\section{Relationship between CYP2D6 polymorphisms and adverse effects}

The relationships between CYP2D6 polymorphisms and adverse effects are shown in Table 3 . The change in weight from baseline to week 4 was significantly different between genotypes $(F=7.514, P=0.001)$. Post-hoc tests for pairwise comparisons between the various genotypes identified significant differences between subjects with the CYP2D6*10 and CYP2D6*65 alleles $(P<$ 0.001 ), and between those with the CYP2D6*65 and CYP2D6*2 $(P=0.025)$ alleles. The differences between HDL from baseline to week 8 were also significantly different between genotypes $(F=3.366, P=0.042)$, and persisted between individuals with the CYP2D6*10 and $C Y P 2 D 6 * 65$ alleles, when between-group comparisons were conducted $(P=0.012)$. Prolactin levels were significantly associated with genotype $(F=4.359, P=0.017)$. Post-hoc pairwise comparisons indicated that the change in prolactin levels in subjects with the CYP2D6*65 allele was significantly higher than other genotypes from baseline to week 8 , especially the $C Y P 2 D 6 * 10$ allele (both

Table 2 Relationships between CYP2D6 genotype and clinical outcomes

\begin{tabular}{|c|c|c|c|c|c|}
\hline & \multicolumn{5}{|c|}{ Total study population $(n=76)$} \\
\hline & CYP2D6*10 & CYP2D6*2 & $C Y P 2 D 6 * 65$ & Statistics & $P$ \\
\hline PANSSO - week 4 & $19.0(12.0,29.0)$ & $24.0(13.0,21.0)$ & $10.0(7.5,20.8)$ & 5.328 & 0.070 \\
\hline PANSSO - week 8 & $27.5(19.0,33.5)$ & $34.5(31.0,39.5)$ & $17.0(13.0,27.0)$ & 3.850 & 0.027 \\
\hline BPRSO - week 4 & $12.0(8.0,15.0)$ & $12.0(11.0,13.0)$ & $11.5(6.3,13.0)$ & 1.059 & 0.589 \\
\hline BPRSO - week 8 & $16.0(13.0,18.5)$ & $15.0(14.0,18.0)$ & $14.0(7.0,17.0)$ & 1.959 & 0.376 \\
\hline CGI-SO - week 4 & $1.0(1.0,2.0)$ & $2.0(1.0,2.0)$ & $1.0(1.0,1.0)$ & 2.853 & 0.240 \\
\hline CGI-SO - week 8 & $2.0(2.0,3.0)$ & $3.0(2.0,4.0)$ & $2.0(2.0,3.0)$ & 1.701 & 0.427 \\
\hline CGI-I2 - week 4 & $0.0(0.0,1.0)$ & $0.0(0.0,0.0)$ & $1.0(0.0,1.0)$ & 2.523 & 0.283 \\
\hline CGI-I2 - week 8 & $1.0(0.0,1.0)$ & $1.0(0.0,2.0)$ & $1.0(0.0,1.5)$ & 1.249 & 0.536 \\
\hline
\end{tabular}

Data are presented as median (interquartile range)

PANSSO - week 4 represents the change of PANSS score from 0 to 4 weeks

Abbreviations: BPRS Brief Psychiatric Rating Scale, CGI Clinical Global Impression, CGI-S Clinical Global Impression Severity, CGI-I Clinical Global Impression Improvement, PANSS Positive and Negative Syndrome Scale 
Table 3 Relationships between genotype and adverse effects

\begin{tabular}{|c|c|c|c|c|c|}
\hline & CYP2D6*10 & CYP2D6*2 & CYP2D6*65 & Statistics & $P$ \\
\hline Weight change from week 0 to $4, \mathrm{~kg}$ & $-1.2 \pm 3.0$ & $-2.6 \pm 2.5$ & $-13.8 \pm 23.4$ & 7.514 & 0.001 \\
\hline Weight change from week 0 to $8, \mathrm{~kg}$ & $-1.6 \pm 3.6$ & $-3.4 \pm 2.0$ & $-1.3 \pm 3.8$ & 0.798 & 0.455 \\
\hline BMI change from week 0 to 8 & $-0.6 \pm 1.3$ & $-1.3 \pm 0.8$ & $-0.5 \pm 1.3$ & 0.985 & 0.380 \\
\hline GLU change from week 0 to $4, \mathrm{mmol} / \mathrm{l}$ & $-0.5 \pm 1.8$ & $-0.3 \pm 0.4$ & $-0.2 \pm 2.8$ & 0.102 & 0.903 \\
\hline GLU change from week 0 to $8, \mathrm{mmol} / \mathrm{l}$ & $-0.3 \pm 0.8$ & $-0.6 \pm 0.3$ & $0.0 \pm 1.4$ & 0.596 & 0.554 \\
\hline TG change from week 0 to $4, \mathrm{mmol} / \mathrm{l}$ & $0.0 \pm 1.0$ & $0.4 \pm 1.1$ & $-0.2 \pm 0.6$ & 0.994 & 0.376 \\
\hline TG change from week 0 to $8, \mathrm{mmol} / \mathrm{l}$ & $-0.1 \pm 1.0$ & $-0.7 \pm 1.6$ & $-0.3 \pm 0.7$ & 1.800 & 0.175 \\
\hline $\mathrm{CHO}$ change from week 0 to $4, \mathrm{mmol} / \mathrm{l}$ & $-0.3 \pm 0.7$ & $-0.1 \pm 0.8$ & $-0.6 \pm 1.1$ & 0.984 & 0.379 \\
\hline $\mathrm{CHO}$ change from week 0 to $8, \mathrm{mmol} / \mathrm{l}$ & $-0.2 \pm 0.8$ & $-0.2 \pm 0.8$ & $-1.0 \pm 2.0$ & 2.395 & 0.100 \\
\hline LDL change from week 0 to $4, \mathrm{mmol} / \mathrm{l}$ & $-0.1 \pm 0.5$ & $-0.1 \pm 0.8$ & $-0.2 \pm 0.5$ & 0.964 & 0.387 \\
\hline LDL change from week0 to $8, \mathrm{mmol} / / \mathrm{l}$ & $-0.1 \pm 0.7$ & $-0.1 \pm 0.2$ & $-0.5 \pm 0.7$ & 2.631 & 0.081 \\
\hline $\mathrm{HDL}$ change from week 0 to $4, \mathrm{mmol} / \mathrm{l}$ & $-0.1 \pm 0.2$ & $-0.1 \pm 0.2$ & $-0.2 \pm 0.2$ & 1.041 & 0.359 \\
\hline $\mathrm{HDL}$ change from week 0 to $8, \mathrm{mmol} / \mathrm{l}$ & $0.0 \pm 0.2$ & $-0.1 \pm 0.2$ & $-0.3 \pm 0.5$ & 3.366 & 0.042 \\
\hline PRL change from week 0 to $4, \mathrm{mmol} / \mathrm{l}$ & $52.1 \pm 62.9$ & $63.4 \pm 63.3$ & $72.5 \pm 79.8$ & 0.509 & 0.603 \\
\hline PRL change from week 0 to $8, \mathrm{mmol} / \mathrm{l}$ & $39.4 \pm 53.7$ & $80.0 \pm 65.8$ & $95.4 \pm 76.1$ & 4.359 & 0.017 \\
\hline ESRS week 4 & 8 & 0 & 5 & 5.858 & 0.037 \\
\hline ESRS week 8 & 11 & 1 & 4 & 0.930 & 0.633 \\
\hline BAS week 4 & 5 & 1 & 2 & 0.897 & 0.712 \\
\hline BAS week 8 & 6 & 1 & 1 & 0.493 & 0.632 \\
\hline
\end{tabular}

Data are presented as mean \pm standard deviation. Abbreviations: BAS Barnes Akathisia Scale, BMI Body mass index, CHO Cholesterol, ESRS Extrapyramidal Symptom Rating Scale, GLU Glucose, HDL High-density lipoprotein, LDL Low-density lipoprotein, PRL Prolactin, TG Triglyceride

$P<0.05)$. The occurrence of EPS was significantly different between different groups $(F=5.858, p=0.037)$, which is mainly attributed to the higher occurrence of EPS in individuals with the CYP2D6*10 allele compared with the other two genotypes.

\section{Differences in plasma concentration of risperidone and response to treatment between subjects with} heterozygous or homozygous mutations of CYP2D6*10 Of the 47 subjects who carried the CYP2D6*10 allele, 26 exhibited homozygous (TT) mutations, while the remaining 21 carried heterozygous mutations (CT). The plasma concentration of risperidone was significantly higher in subjects with homozygous mutations of CYP2D6*10 compared with heterozygous mutations, at each time point (Fig. 2; all $P<0.01$ ). The difference in $\mathrm{R} / 9-\mathrm{OH}$ ratio was also significant at each time point (all $P<0.01$ ), while 9-OH-RIS was only significantly different at week $2(Z=-1.988, P=0.046)$. The C/D ratio was significantly different between CYP2D6*10TT/CT carriers at weeks 4 and $8(Z=-2.274$, $P=0.025$ and $Z=-2.155, P=0.031$, respectively) but not at week 2 . The change in the BPRS score from baseline to week 8 was significantly different between subjects with heterozygous or homozygous mutations $(Z=4.667$,
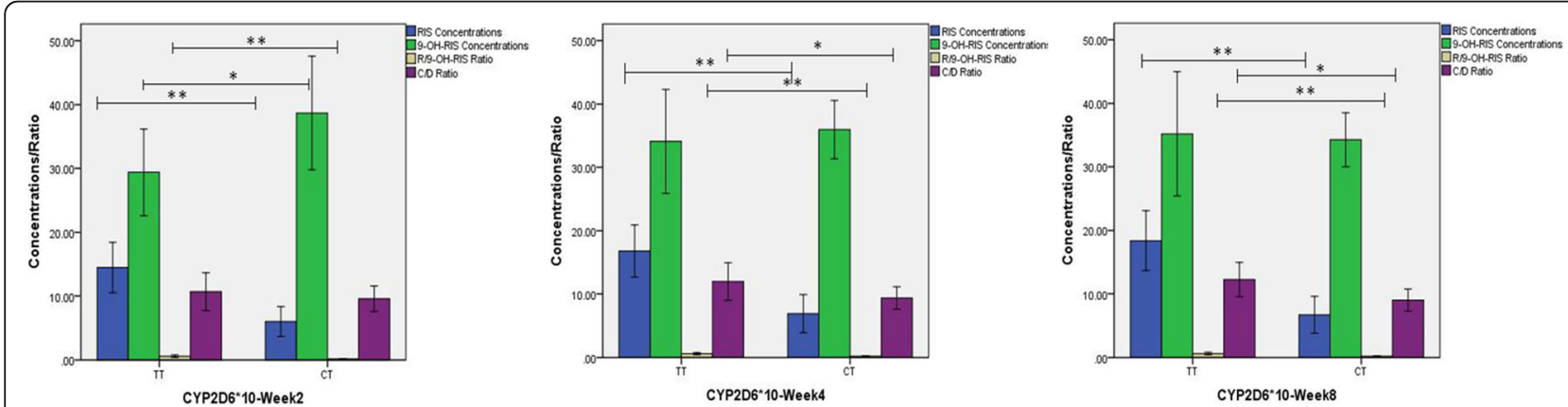

Fig. 2 Bar graphs showing plasma concentrations of risperidone in subjects with heterozygous and homozygous mutations of CYP2D6*10. Abbreviations: C/D, concentration/dose ratio; RIS, risperidone; 9-OH-RIS, 9-hydroxyrisperidone; R/9-OH-RIS Ratio, risperidone/9-hydroxyrisperidone ratio; ${ }^{*} p<0.05$ 
$P=0.040$ ) (Table 4). We did not observe any significant differences in adverse effects between subjects with either mutation (all $P>0.05$ ) (Table S2).

\section{Single nucleotide polymorphisms, plasma concentration of risperidone, clinical response, and adverse effects in relation to polymorphisms of C100T, C2851T, and G4181C Demographic, clinical, and genetic characteristics}

The demographics and disease-specific characteristics of subjects are stratified by single nucleotide polymorphism (SNP; i.e., C100T, C2851T, or G4181C) and presented in Table S3. The mean age was significantly higher in subjects with the $G 4181 C$ polymorphism in the $\mathrm{C} / \mathrm{C}$ genotype $(F=-2.982, P=0.003)$. No other significant differences were identified in age or gender between the polymorphisms (both $P>0.05$ ). Additionally, there were no significant differences in plasma concentration of risperidone, PANSS, BPRS, or CGI at baseline between subjects with different polymorphisms (all $P>0.05)$.

\section{Plasma concentration of risperidone in relation to single- nucleotide polymorphism}

Figure 3 illustrates the plasma concentration of risperidone with relation to the subjects respective SNP. Plasma concentrations of risperidone and the $\mathrm{R} / 9-\mathrm{OH}$ ratio were significantly higher in subjects with the $\mathrm{T} / \mathrm{T}$ genotype compared with other genotypes in C100T at each time point (all $P<0.05$ ). Further pairwise comparisons identified no other significant difference between the $\mathrm{C} / \mathrm{C}$ and $\mathrm{C} / \mathrm{T}$ genotypes in subjects with $C 100 T(P>$ $0.05)$. In terms of $G 4181 C$, a significantly higher plasma concentration of risperidone was identified in the $\mathrm{C} / \mathrm{C}$ genotype compared with the $\mathrm{G} / \mathrm{C}$ genotype at each time point (all $P<0.05$ ). However, the 9-OH-RIS was not significantly different between genotypes in both C100T and $G 4181 C$ at any time point (all $P>0.05$ ). The C/D

Table 4 Relationships between response to treatment and CYP2D6*10 allelic mutation

\begin{tabular}{lllll}
\hline & TT (26) & CT (21) & Statistics & $\boldsymbol{P}$ \\
\hline PANSSO - week 4 & $20.5(12.0,28.2)$ & $19.0(10.5,35.0)$ & 1.357 & 0.645 \\
PANSS0 - week 8 & $27.0(19.0,32.0)$ & $30.0(17.0,39.5)$ & 4.287 & 0.235 \\
BPRSO - week4 & $34.0(28.8,40.0)$ & $32.5(30.8,34.2)$ & 15.136 & 0.291 \\
BPRSO - week8 & $29.0(27.0,34.0)$ & $28.0(26.3,30.0)$ & 4.667 & 0.040 \\
CGI-S0 - week4 & $4.0(3.0,4.0)$ & $3.5(3.0,4.0)$ & -1.392 & 0.164 \\
CGI-S0 - week 8 & $3.0(2.0,3.0)$ & $2.0(2.0,3.0)$ & -0.125 & 0.900 \\
CGI-I2 - week 4 & $-0.5(-1.0,0.0)$ & $0.0(-1.0,0.0)$ & -0.599 & 0.549 \\
CGI-I2 - week 8 & $0.0(-1.0,1.0)$ & $0.0(-1.0,1.0)$ & -3.84 & 0.701 \\
\hline
\end{tabular}

Data are presented as median (interquartile range)

PANSSO - week 4 represents the change of PANSS score from 0 to 4 weeks Abbreviations: BPRS Brief Psychiatric Rating Scale, CGI Clinical Global Impression, CGI-S Clinical Global Impression Severity, CGI-I Clinical Global Impression Improvement PANSS Positive and Negative Syndrome Scale ratio was significantly different between subjects with C100T and $G 4181 C$ polymorphisms (all $P<0.05$ ) and between subjects with $\mathrm{CC} / \mathrm{TT}$ and $\mathrm{CT} / \mathrm{TT}$ genotypes in further pairwise comparisons at any time point (all $P<$ 0.05). For the $C 2851 T$ polymorphism, plasma drug concentration, $\mathrm{R} / 9-\mathrm{OH}$, or $\mathrm{C} / \mathrm{D}$ ratio were not significantly different for any genotype (all $P>0.05$ ).

\section{Clinical response and adverse effects in relation to single- nucleotide polymorphism}

The associations between SNPs and clinical responses to risperidone treatment are presented in Table S4; there was no apparent association between improvement in PANSS, BPRS, or CGI scores at week 4 or 8 in relation to C100T and C2851T polymorphisms (all $P>0.05$ ). However, for the G4181C polymorphism, improvement in CGI-S and CGI-I scores at week 4 were significantly different between the $\mathrm{C} / \mathrm{C}$ and $\mathrm{C} / \mathrm{G}$ genotypes $(\mathrm{Z}=$ 1.118, $P=0.016$ and $Z=-2.586, P=0.010$, respectively). Analysis of the predictive values of the alleles (CYP2D6*10, CYP2D6*2, or CYP2D6*65,) and SNPs (C100T, C2851T, and G4181C) with regard to clinical response (defined as $25 \%$ improvement in PANSS from baseline), identified 30 and 40 subjects as treatment responders at weeks 4 and 8 , respectively. After controlling for confounding factors, regression analysis showed that plasma concentrations of risperidone at week 2 were a statistically significant predictor of clinical response $(B=$ $0.642, P=0.020$ for response at week $4, B=0.946, P=$ 0.003 for response at week 8 ). The relationship between adverse effects and SNPs are shown in Table 5. There were no significant differences in incidence of adverse metabolic effects or EPS between genotypes of the C100T polymorphism (all $P>0.05$ ). In patients with the $C 2851 T$ polymorphism, adverse metabolic effects, including weight change from baseline to week $4(\mathrm{Z}=$ 3.091, $P=0.008$ ), changes in HDL from baseline to week $4(\mathrm{Z}=-2.073, P=0.038)$ and week $8(\mathrm{Z}=4.061, P=$ $0.035)$, and change in PRL from baseline to week 8 $(\mathrm{Z}=-2.179, P=0.029)$, were significantly higher among patients with the $C / T$ genotype compared with the $C / C$ genotype. In contrast, the incidence of EPS, as assessed by ESRS or BAS, did not differ significantly in relation to genotype. With regards to the G4181C polymorphism, the change in GLU from baseline to week $4(\mathrm{Z}=-2.401$ $P=0.016)$ and the TG level from baseline to week 4 $(\mathrm{Z}=3.206, P=0.001)$, were significantly higher in subjects with the $\mathrm{C} / \mathrm{C}$ genotype compared with $\mathrm{C} / \mathrm{G}$, however, by week 8 , this difference was not statistically significant $(Z=-0.824, P=0.410)$. There were no significant differences in any other adverse metabolic effects or the incidence of EPS, between genotypes of the G4181C polymorphism (all $P>0.05$ ). 

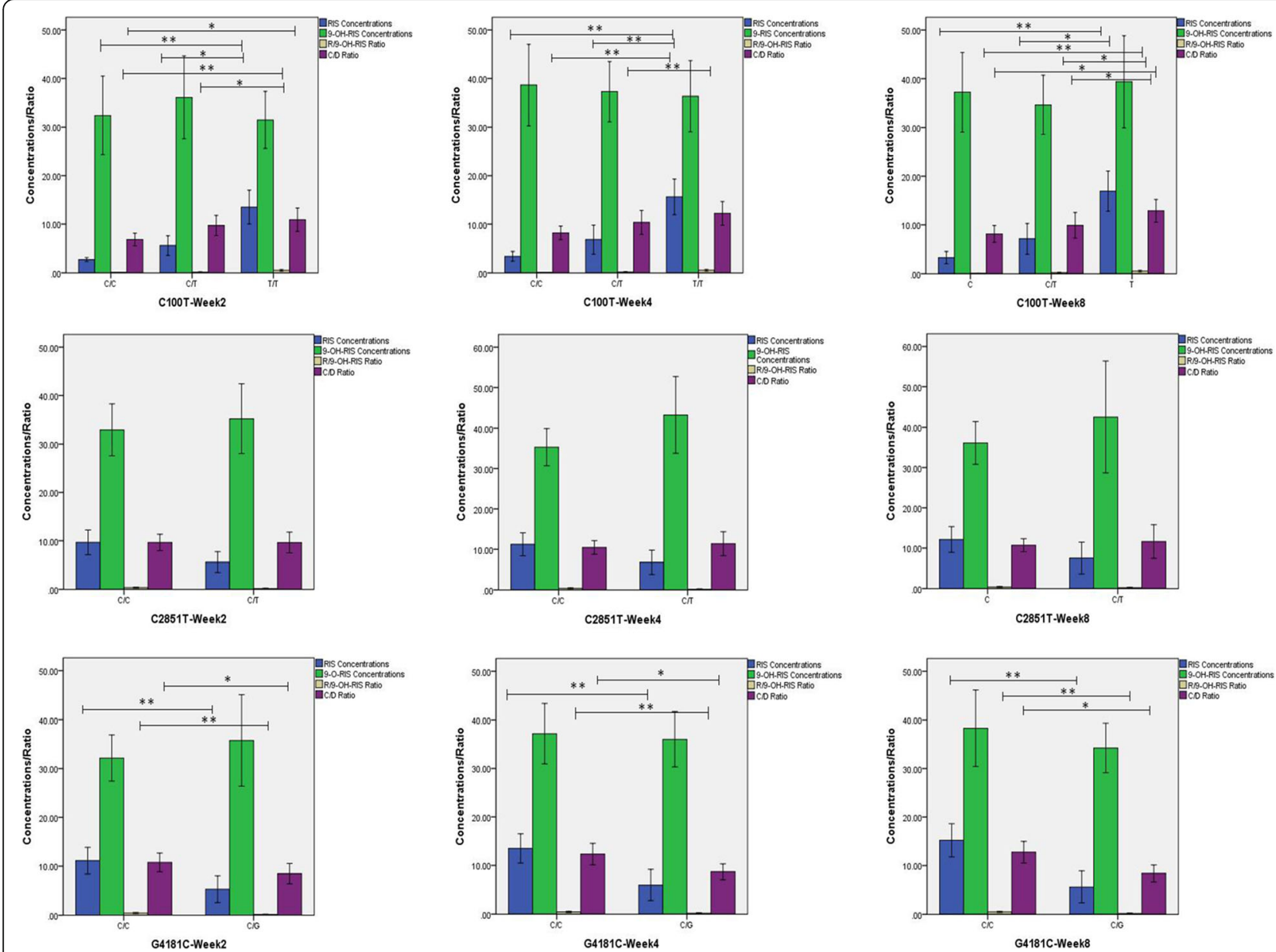

Fig. 3 Plasma concentrations of risperidone in relation to polymorphisms of C100T, C2851T, and G4181C. Abbreviations: C/D, concentration/dose ratio; RIS, risperidone; 9-OH-RIS, 9-hydroxyrisperidone; R/9-OH-RIS Ratio, risperidone/9-hydroxyrisperidone ratio; ${ }^{*} p<0.05$

\section{Discussion}

The present study demonstrates that among patients with schizophrenia, the CYP2D6 genotype significantly influenced the plasma concentration of risperidone. This finding also suggests that among patients treated with risperidone, genotype may influence adverse drug reactions. However, the CYP2D6 genotype exerts a slight effect on improvement of clinical symptoms.

The main finding of the current study is that the plasma drug concentrations of risperidone were significantly different among different CYP2D6 alleles or SNPs. Similarly, significant differences were also observed between heterozygotes and homozygotes among the most common $C Y P 2 D 6 * 10$ alleles. Different CYP2D6 alleles or SNPs were significantly associated with adverse drug effects, including adverse metabolic reactions and EPS. No obvious differences were observed between heterozygotes and homozygotes among the CYP2D6*10 allele. Only a slight difference was reported in the improvement of clinical symptoms among individuals with different CYP2D6 genotypes.
Our study revealed that the CYP2D6 genotype influenced the metabolism of risperidone, evidenced by increased plasma concentration and metabolic ratio (RIS/ 9-OH-RIS) [12, 16, 22]. Furthermore, our investigation of different alleles agree with the results of previous studies [12, 23], as well as a recent study involving children and adolescents with autism spectrum disorders which reported plasma concentrations of risperidone and RIS/9-OH-RIS to be significantly associated with CYP2D6*10, while plasma concentration of 9-OH-RIS was not found to be significantly correlated [13]. However, some studies have shown CYP2D6*10 to be associated with plasma concentrations of both risperidone and 9-OH-RIS, conflicting with our results [22, 23].

We found that after treatment, the plasma concentration of risperidone, was significantly different depending on the homo- or heterozygosity of CYP2D6*10 mutations, confirming previous studies that reported subjects who had homozygous mutations $C Y P 2 D 6^{*} 10$ to have higher plasma concentrations of risperidone compared with single-allele carriers $[24,25]$. This may be attributed to 


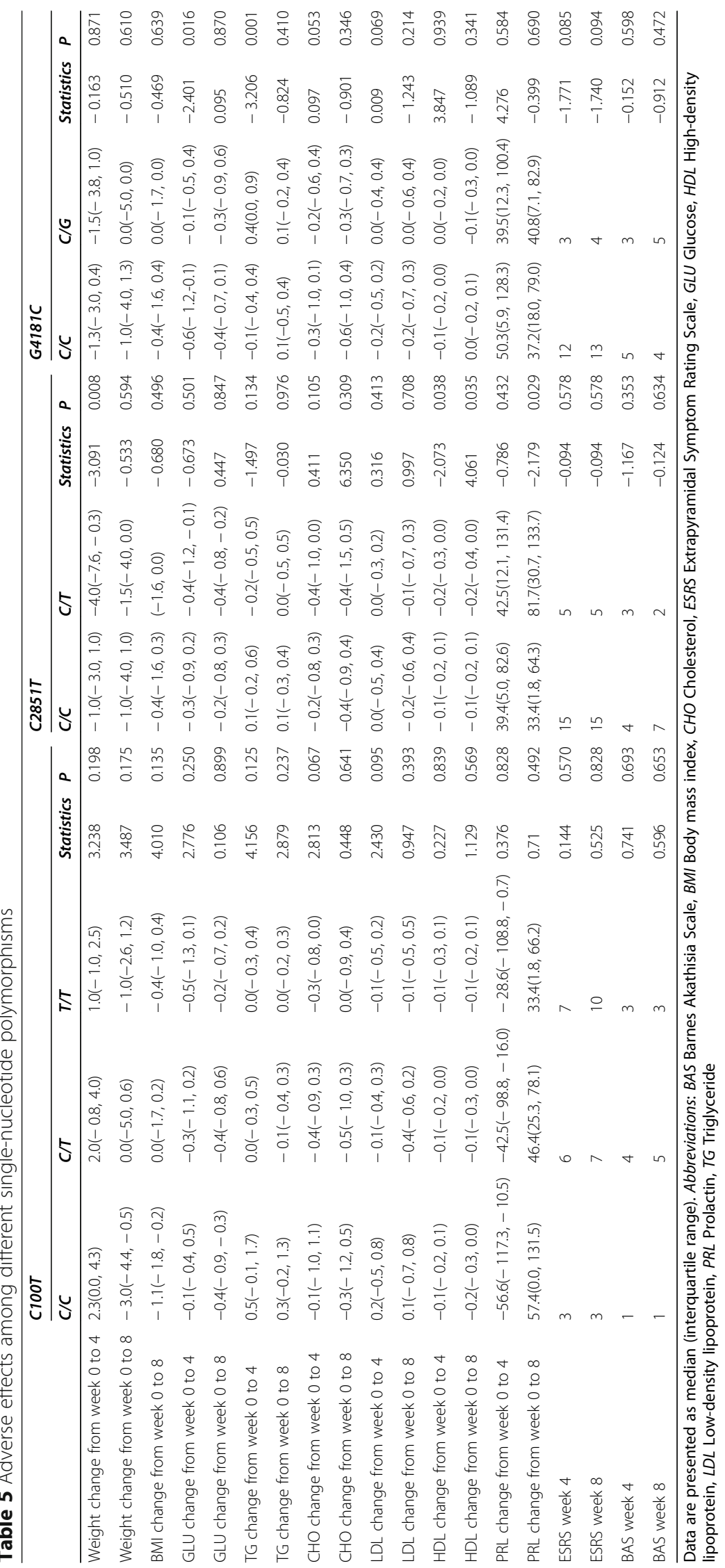


differences in enzymatic activity; the enzymatic activity of the CYP2D6 gene product is significantly lower in CYP2D6*10-homozygous subjects compared with wildtype homozygotes, while the CYP2D6*10-heterozygous subjects exhibit moderate enzyme activity [26].. Therefore, CYP2D6*10 is associated with lower enzymatic activity. Our study revealed that subjects with $C Y P 2 D 6 * 2$ have lower plasma concentrations of risperidone, indicating increased enzymatic activity after treatment, compared with the other two genotypes. Thus, there appears to be a significant relationship between CYP2D6 genotype and CYP2D6 activity; however, to the best of our knowledge, no study has directly compared CYP2D6 activity between these three genotypes. Indeed, research regarding CYP2D6*65 and CYP2D6*2 is limited. Considering the small sample size of the present study, the differences that we have identified should be considered preliminary and interpreted with caution; further long-term large-scale research is warranted.

Significant associations were observed between SNPs (C100T, C2851T, and G4181C) and plasma concentrations of risperidone, RIS/9-OH-RIS ratio, and C/D ratio. To the best of our knowledge, this is the first exploratory research to investigate the relationships between CYP2D6 SNPs, plasma concentration of antipsychotics, and clinical response relative to antipsychotic treatment, in patients with schizophrenia. Similar research involving psychiatric patients has shown the CYP2D6 1584C > $\mathrm{G}$ polymorphism as having a significant influence on thioridazine:mesoridazine plasma ratio, although the correlation between polymorphisms and clinical response was not investigated in this study [27]. We discovered that the C100T and G4181C polymorphisms were associated with differences in plasma concentration of risperidone and RIS/9-OH-RIS ratio. These findings indicate that even single-nucleotide mutations are sufficient to affect the activity of metabolic enzymes. Furthermore, the C/D ratio was obviously different between SNPs, suggesting that plasma clearance varies according to allelic variants. These factors may contribute to the clinical treatment response, and these findings provide new insight for individualized drug therapy.

We found CYP2D6*2 to be associated with increased improvement in clinical symptom, while CYP2D6*65 was found to be more associated with metabolic indicators. These observations contradict our hypothesis that carriers of $C Y P 2 D 6^{*} 2$ have higher enzymatic activity of $C Y P 2 D 6$, resulting in reduced plasma concentration of risperidone and therefore, reduced improvement of clinical symptoms. These findings are also inconsistent with previous research which has demonstrated lower enzymatic activity to be associated with an increased clinical response to risperidone treatment [14]. This contradiction may be attributed to the smaller sample size with the CYP2D6*2 allele. We did not detect a significant association between $C Y P 2 D 6 * 10$ and improvement of clinical symptoms, confirming the results of previous studies [28]. However, investigations regarding the relationships between genotype and clinical response are scarce and discrepant; studies involving psychiatric patients receiving risperidone have not identified any association between CYP2D6 polymorphism and clinical improvement [15, 16]. Besides, it is difficult to compare studies including subjects with various combinations of genotypes $[15,16]$. With regards to SNPs, we did not identify any significant differences in clinical response among the different single nucleotide variants, contrary to our hypothesis. The small sample size may have contributed to the lack of clarity, which is exacerbated by the lack of similar studies. Further studies with larger sample sizes are warranted to clarify the relationship between SNPs and clinical response.

Consistent with previous research, we found clear associations between genotype and adverse effects, including adverse metabolic reactions and EPS. A study involving children and adolescents receiving antipsychotics identified a significant difference in weight gain between patients with the CYP2D6"1/*4 genotype and who did not carry allele*4 [29]. Another study reported that patients with the CYP2D6 "1/*3 or *4 genotype, that were treated with atypical antipsychotics exhibited significantly larger percent change in body mass index $(p<$ $0.0097)$ compared with those with a "1/"1 genotype [30]. In the present research, CYP2D6*10 and CYP2D6*2 were found to be associated with increased weight gain compared with $C Y P 2 D 6 * 65$, which contradicts some previous reports but agrees with others. A previous study suggested that individuals with the poor-metabolizer phenotype have increased plasma drug levels after treatment, resulting in serious antipsychotic-induced toxicity with consequent dose-dependent complications [31]. Another study confirmed that the poor-metabolizer phenotype was associated with an increased incidence of adverse effects of risperidone treatment [19]. Theoretically, CYP2D6*65 should be related to increased weight again while $C Y P 2 D 6 * 2$ should not be. Lane et al. reported a significant correlation between CYP2D6*10 and weight gain in patients receiving risperidone [32]. These findings are supported by the results of the present study, which also demonstrated a significant difference in the change of HDL from baseline in relation to genotype; CYP2D6"65 was associated with lower HDL levels following risperidone treatment compared with the other genotypes. The relationship between HDL and CYP2D6 genotype has not been reported previously. In contrast, we found that in response to risperidone treatment, CYP2D6*65 was associated with higher levels of prolactin compared with the other alleles, particularly CYP2D6*10. Previous reports on the association between 
CYP2D6 polymorphisms in relation to prolactin concentrations, are controversial. One study explored the impact of CYP2D6 polymorphisms on the prevalence of risperidone-induced adverse effects, including hyperprolactinemia, revealed that the poor-metabolizer phenotype influenced the frequency of adverse effects and poor treatment compliance [19]. Although only assessed in a small cohort, another study revealed that CYP2D6 ultra-rapid metabolism may contribute to increased prolactin levels in children, consist with our observations [33]. Also consistent with the results of a previous study [34], we found that the incidence of EPS was significantly higher among subjects with the CYP2D6"10 genotype. However, the predictive value of genotype for extrapyramidal side-effects is controversial [31, 35]. Some authors have suggested that "poor metabolizers" have significantly more pronounced or severe predisposing factors for the development of acute EPS [19, 34], which is supported by our finding that subjects carrying CYP2D6*10 or CYP2D6*65 are more likely to suffer EPS. With respect to SNPs, our study revealed that weight change and HDL or PRL alterations from baseline in response to treatment, differed significantly between subjects with C2851T wild-type compared with mutant, while GLU and TG differed significantly between subjects with $\mathrm{G} 4181 \mathrm{C}$ homozygous mutations compared with heterozygous mutations. Previous studies on SNPs and their association with adverse reactions are scarce, with only one published report describing the association between the $1846 \mathrm{G}>\mathrm{A}$ polymorphism of CYP2D6 and extrapyramidal side effects [36]. Our results suggest that SNPs affect the clinical response, and occurrence of adverse reactions, to risperidone treatment. These data reveal a potential tool for predicting treatment efficacy and may also facilitate the prevention of adverse effects.

Collectively, the current study showed that polymorphisms of the CYP2D6 gene had a significant effect on drug concentration and adverse drug effects. Previous studies have shown that polymorphisms of the CYP2D6 gene display different enzyme activity including decreased, increased, or nonfunctional enzymatic activity [37]. Different enzymatic activity contributed to interindividual variability in plasma drug concentration, and subsequently, clinical outcomes [10]. Our study did reveal that poorly metabolizing genotypes, such as CYP2D6*10, were associated with more severe adverse effects, including weight gain and increased HDL and EPS, supporting the assumption that CYP2D6 gene polymorphisms may be associated with different adverse reactions via alterations in enzyme activity. However, the slight improvement in clinical symptoms did not seem to be associated with CYP2D6 genetic variations.

The present study has some limitations which should be acknowledged. Firstly, the sample size was small.
Secondly, subjects were followed-up for only 8 weeks; therefore, we cannot comment on the effects of CYP2D6 polymorphisms on the long-term clinical responses and side-effects, which may affect our conclusions. Additionally, patients who were non-compliant with treatment were excluded from the analysis and the study could be missing some key patient characteristics that influence drug treatment response. Finally, we only identified three significant single-nucleotide gene-effective mutation sites. Therefore, comparisons between more mutation sites could not be performed. Further verification through studies involving large sample sizes and comparing more genotypes are warranted.

\section{Conclusion}

In summary, our study reveals the association between CYP2D6 polymorphisms and the plasma concentration of risperidone, and that CYP2D6 genotypes may serve as a predictor of adverse reactions, but not improvement of clinical symptoms, after risperidone treatment.

\section{Supplementary Information}

The online version contains supplementary material available at https://doi. org/10.1186/s12888-020-03034-9.

\section{Additional file 1.}

\section{Abbreviations}

BMI: Body mass index; PANSS: The Positive and Negative Syndrome Scale; BPRS: Brief Psychiatric Rating Scale; CGI-S: Clinical Global Impression-Severity; CGI-I: Clinical Global Impression-Improvement; BAS: Barnes Akathisia Scale; CHO: Cholesterol; ESRS: Extrapyramidal Symptom Rating Scale; GLU: Glucose; HDL: High-density lipoprotein; LDL: Low-density lipoprotein; PRL: Prolactin; TG: Triglyceride; C/D: Concentration/dose ratio; RIS: Risperidone; 9-OH-RIS: 9hydroxyrisperidone; R/9-OH-RIS Ratio: Risperidone/9-hydroxyrisperidone ratio

\section{Acknowledgements \\ None.}

\section{Authors' contributions}

YY1 and $J L$ contributed equally to this paper. JL contributed to data collection and $Y Y 1$ analyzed data and drafted and edited the manuscript. JL, $Z W, Y H, Y Y 2, K F, W J, Y X, W L$ and JZ helped recruit trial participants.RW organized the database and supervised the entire project. All authors reviewed, edited, contributed, and approved the final version of the manuscript.

\section{Funding}

This research is support by a program grant from the Wuxi Health and Family Planning Commission (MS201707). The Wuxi Health and Family Planning Commission had no role in the design of the study, data collection, analysis, data interpretation, or in writing the manuscript.

\section{Availability of data and materials}

The datasets used are available from the corresponding author on reasonable request.

\section{Ethics approval and consent to participate}

This study was approved by the Ethics Committee of the third people's Hospital of Jiangyin City on August 28, 2017. All participants signed consent prior to participation and were allowed to stop participation without prejudice. 


\section{Consent for publication}

Not applicable.

\section{Competing interests}

The authors declare no conflict of interest.

Received: 11 August 2020 Accepted: 29 December 2020 Published online: 03 February 2021

\section{References}

1. Leucht S, Cipriani A, Spineli L, Mavridis D, Örey D, Richter F, et al. Comparative efficacy and tolerability of 15 antipsychotic drugs in schizophrenia: a multiple-treatments meta-analysis. Lancet. 2013;382(9896): 951-62.

2. Lieberman JA, Stroup TS, McEvoy JP, Swartz MS, Rosenheck RA, Perkins DO, et al. Effectiveness of antipsychotic drugs in patients with chronic schizophrenia. N Engl J Med. 2005;353(12):1209-23.

3. Yoshimura Y, Takeda T, Kishi Y, Harada T, Nomura A, Washida K, et al. Twoyear effectiveness of risperidone and aripiprazole in the maintenance treatment of patients with recent-onset or chronic schizophrenia and related psychotic disorders: a retrospective multicenter study. Int Clin Psychopharmacol. 2018;33(3):147-54.

4. Grunder G, Hiemke C, Paulzen M, Veselinovic T, Vernaleken I. Therapeutic plasma concentrations of antidepressants and antipsychotics: lessons from PET imaging. Pharmacopsychiatry. 2011;44(6):236-48.

5. Kapur S, Zipursky RB, Remington G. Clinical and theoretical implications of 5-HT2 and D2 receptor occupancy of clozapine, risperidone, and olanzapine in schizophrenia. Am J Psychiatry. 1999;156:286-93.

6. LLerena A, Berecz R, Peñas-Lledó E, Süveges A, Fariñas H. Pharmacogenetics of clinical response to risperidone. Pharmacogenomics. 2013;14(2):177-94.

7. Hiemke C, Bergemann N, Clement HW, Conca A, Deckert J, Domschke K, et al. Consensus guidelines for therapeutic drug monitoring in neuropsychopharmacology: Update 2017. Pharmacopsychiatry. 2018:51(102):9-62.

8. Spina E, de Leon J. Clinical applications of CYP genotyping in psychiatry. J Neural Transm (Vienna). 2015:122(1):5-28.

9. de Leon J, Wynn G, Sandson NB. The pharmacokinetics of paliperidone versus risperidone. Psychosomatics. 2010:51(1):80-8.

10. Cartwright AL, Wilby KJ, Corrigan S, Ensom MH. Pharmacogenetics of risperidone: a systematic review of the clinical effects of CYP2D6 polymorphisms. Ann Pharmacother. 2013:47(3):350-60.

11. Puangpetch A, Vanwong N, Nuntamool N, Hongkaew Y, Chamnanphon M Sukasem C. CYP2D6 polymorphisms and their influence on risperidone treatment. Pharmgenomics Pers Med. 2016;9:131-47.

12. Xiang Q, Zhao X, Zhou Y, Duan JL, Cui YM. Effect of CYP2D6, CYP3A5, and MDR1 genetic polymorphisms on the pharmacokinetics of risperidone and its active moiety. J Clin Pharmacol. 2010;50(6):659-66.

13. Vanwong N, Ngamsamut N, Medhasi S, Puangpetch A, Chamnanphon M, Tan-Kam T, et al. Impact of CYP2D6 polymorphism on steady-state plasma levels of risperidone and 9-hydroxyrisperidone in Thai children and adolescents with autism spectrum disorder. J Child Adolesc Psychopharmacol. 2017;27(2):185-91.

14. Almoguera B, Riveiro-Alvarez R, Lopez-Castroman J, Dorado P, VaqueroLorenzo C, Fernandez-Piqueras J, et al. CYP2D6 poor metabolizer status might be associated with better response to risperidone treatment. Pharmacogenet Genomics. 2013;23(11):627-30.

15. Kakihara S, Yoshimura R, Shinkai K, Matsumoto C, Goto M, Kaji K, et al. Prediction of response to risperidone treatment with respect to plasma concencentrations of risperidone, catecholamine metabolites, and polymorphism of cytochrome P450 2D6. Int Clin Psychopharmacol. 2005;20:71-8.

16. Jovanović $N$, Božina $N$, Lovrić $M$, Medved V, Jakovljević M, Peleš AM. The role of CYP2D6 and ABCB1 pharmacogenetics in drug-naïve patients with first-episode schizophrenia treated with risperidone. Eur I Clin Pharmacol. 2010;66(11):1109-17.

17. Nasrallah HA. Metabolic findings from the CATIE trial and their relation to tolerability. CNS Spectr. 2006;11(7 Suppl 7):32-9.

18. Roke Y, van Harten PN, Franke B, Galesloot TE, Boot AM, Buitelaar JK. The effect of the Taq1A variant in the dopamine $D(2)$ receptor gene and common CYP2D6 alleles on prolactin levels in risperidone-treated boys. Pharmacogenet Genomics. 2013;23(9):487-93.
19. de Leon JSM, Pan RM, Fairchild M, Koch WH, Wedlund PJ. The CYP2D6 poor metabolizer phenotype may be associated with risperidone adverse drug reactions and discontinuation. J Clin Psychiatry. 2005;66:15-27.

20. Cabaleiro T, Ochoa D, Lopez-Rodriguez R, Roman M, Novalbos J, Ayuso C, et al. Effect of polymorphisms on the pharmacokinetics, pharmacodynamics, and safety of risperidone in healthy volunteers. Hum Psychopharmacol. 2014;29(5):459-69.

21. Božina N, Jovanovic N, Lovric M, Medved V. Clinical significance of a CYP2D6 poor metabolizer-a patient with schizophrenia on risperidone treatment. Ther Drug Monit. 2008;30:748-51.

22. Yoo HD, Lee SN, Kang HA, Cho HY, Lee IK, Lee YB. Influence of ABCB1 genetic polymorphisms on the pharmacokinetics of risperidone in healthy subjects with CYP2D6*10/*10. Br J Pharmacol. 2011;164(2b):433-43.

23. Cho HY, Lee YB. Pharmacokinetics and bioequivalence evaluation of risperidone in healthy male subjects with different CYP2D6 genotypes. Arch Pharm Res. 2006;29(6):525-33.

24. Suzuki Y, Fukui N, Tsuneyama N, Watanabe J, Ono S, Sugai T, et al. Effect of the cytochrome P450 2D6*10 allele on risperidone metabolism in Japanese psychiatric patients. Hum Psychopharmacol. 2012;27(1):43-6.

25. Mihara K, Kondo T, Yasui-Furukori N, Suzuki A, Ishida M, Ono S, et al. Effects of various CYP2D6 genotypes on the steady-state plasma concentrations of risperidone and its active metabolite, 9-hydroxyrisperidone, in Japanese patients with schizophrenia. Ther Drug Monit. 2003;25:287-93.

26. Johansson I, Oscarson M, Yue QY, Bertilsson L, Sjöqvist F, Ingelman-Sundberg M. Genetic analysis of the Chinese cytochrome P4502D locus: characterization of variant CYP2D6 genes present in subjects with diminished capacity for debrisoquine hydroxylation. Mol Pharmacol. 1994;46:452-9.

27. Dorado P, Peñas-LLedó EM, de la Rubia A, LLerena A. Relevance of CYP2D6 $-1584 C>G$ polymorphism for thioridazine:mesoridazine plasma concentration ratio in psychiatric patients. Pharmacogenomics. 2009;10(7): 1083-9.

28. Kaur G, Gupta D, Chavan BS, Sinhmar V, Prasad R, Tripathi A, et al. Identification of genetic correlates of response to Risperidone: Findings of a multicentric schizophrenia study from India. Asian J Psychiatr. 2017;29:174-82.

29. Nussbaum LA, Dumitraşcu VI, Tudor A, Grădinaru RA, Andreescu N, Puiu M. Molecular study of weight gain related to atypical antipsychotics: clinical implications of the CYP2D6 genotype. Romanian J Morphol Embryol. 2014; 55(3):877-84.

30. Ellingrod VL, Miller D, Schultz SK, Wehring H, Arndt S. CYP2D6 polymorphisms and atypical antipsychotic weight gain. Psychiatr Genet. 2002;12:55-8

31. Ravyn D, Ravyn V, Lowney R, Nasrallah HA. CYP450 pharmacogenetic treatment strategies for antipsychotics: a review of the evidence. Schizophr Res. 2013;149(1-3):1-14.

32. Lane HY, Liu YC, Huang CL, Chang YC, Wu PL, Lu CT, et al. Risperidonerelated weight gain: genetic and nongenetic predictors. J Clin Psychopharmacol. 2006;26(2):128-34.

33. Youngster I, Zachor DA, Gabis LV, Bar-Chaim A, Benveniste-Levkovitz P, Britzi $M$, et al. CYP2D6 genotyping in paediatric patients with autism treated with risperidone: a preliminary cohort study. Dev Med Child Neurol. 2014;56(10): 990-4.

34. Fu Y, Fan CH, Deng HH, Hu SH, Lv DP, Li LH, Wang JJ, et al. Association of CYP2D6 and CYP1A2 gene polymorphism with tardive dyskinesia in Chinese schizophrenic patients. Acta Pharmacol Sin. 2006;27(3):328-32.

35. Plesnicar BK, Zalar B, Breskvar K, Dolzan V. The influence of the CYP2D6 polymorphism on psychopathological and extrapyramidal symptoms in the patients on long-term antipsychotic treatment. J Psychopharmacol. 2006; 20(6):829-33.

36. Sychev DA, Burashnikova IS, Kazakov RE. 1846G >A polymorphism of CYP2D6 gene and extrapyramidal side effects during antipsychotic therapy among Russians and Tatars: a pilot study. Drug Metab Pers Ther. 2016;31(4): 205-12.

37. Ingelman-Sundberg M, Sim SC, Gomez A, Rodriguez-Antona C. Influence of cytochrome P450 polymorphisms on drug therapies: pharmacogenetic, pharmacoepigenetic and clinical aspects. Pharmacol Ther. 2007;116(3):496-526.

\section{Publisher's Note}

Springer Nature remains neutral with regard to jurisdictional claims in published maps and institutional affiliations. 